\title{
Reflux oesophagitis and Helicobacter pylori infection in elderly patients
}

\author{
R Liston, MA Pitt, AK Banerjee
}

\begin{abstract}
Summary
Helicobacter pylori is associated with gastritis, peptic ulcers and gastric malignancies. Little attention has been paid to the possibility that it may also have a role in the pathogenesis of reflux oesophagitis. This is especially true in elderly patients who have life-long infection and provide an ideal group to study the mucosal changes associated with the organism. The aim of this study was to determine if $H$ pylori is associated with reflux oesophagitis in elderly patients. Consecutive gastroscopy patients were recruited. Multiple biopsies were taken from oesophagus, stomach, antrum and duodenum for histology and rapid urease tests. Patients also had IgG ELISA antibodies and ${ }^{13} \mathrm{C}$-urea breath tests performed. Patients with macroscopic or microscopic evidence of reflux oesophagitis were compared to patients with macroscopically normal upper gastrointestinal tracts and no microscopic evidence of reflux. A total of 114 patients were recruited, average age 78.9 years $( \pm 5.4)$. There were 37 refluxers and 33 non-refluxers. We found no evidence for an association between the presence of $H$ pylori and reflux oesophagitis in elderly patients. The high prevalence of $H$ pylori in patients with reflux oesophagitis can be explained by the presence of incidental gastritis.
\end{abstract}

Keywords: Helicobacter pylori, gastritis, reflux oesophagitis, elderly

Bolton General

Hospital, Farnworth,

Bolton BL4 0JR, UK

Department of

Medicine for the

Elderly

R Liston

AK Banerjee

Department of

Histopathology

MA Pitt

Correspondence to Dr R Liston, Clinica Research Fellow,

Department of Geriatric Medicine, Clinical Sciences Building, Hope Hospital, Eccles Old Road, Salford, M6 8HD, UK

Accepted 27 September 1995
The association between Helicobacter pylori, gastritis and peptic ulcer disease is well recognised and duodenal ulceration is now accepted as a curable infectious disease. ${ }^{1,2}$ The importance of $H$ pylori in the pathogenesis of gastric carcinoma and mucosa-associated lymphatic tumours (MALTomas) is also being increasingly recognised. ${ }^{3,4}$ There have also been many studies describing the association of $H$ pylori with gastritis and peptic ulcer disease in elderly patients. ${ }^{5-7}$ Little attention, however, has been paid to the possible role of $H$ pylori in the pathogenesis of reflux oesophagitis and, to our knowledge, no study has specifically looked at this possibility in a group of elderly subjects.

$H$ pylori is probably acquired in childhood and the duration of infection is life-long. ${ }^{8,9} \mathrm{~A}$ large proportion of the elderly population is infected with the organism. ${ }^{10,11}$ These longterm infected elderly patients thus provide an ideal group to study the pathological changes associated with the infection. The aim of this study was to use a wide range of available tests; histology, rapid urease test (CLO test), IgG ELISA antibodies to $H$ pylori and ${ }^{13} \mathrm{C}$-urea breath tests $\left({ }^{13} \mathrm{C}\right.$-UBT) to determine if an association exists between $H$ pylori and reflux oesophagitis in an unselected, consecutive group of elderly patients presenting for routine upper gastrointestinal endoscopy.

\section{Patients and methods}

Consecutive patients admitted for gastroscopy were recruited, regardless of the reason for the procedure. The protocol was passed by the ethics committee of Bolton Hospitals Trust and full informed consent was taken from each patient prior to recruitment. Patients were excluded if they had been on any antibiotic during the preceding three months as it was thought that this may interfere with $H$ pylori detection. ${ }^{7}$ They were also excluded if they had had previous upper gastrointestinal surgery or had been on a proton pump blocker as there is evidence that this medication may also reduce $H$ pylori detection. ${ }^{12}$ Each patient had multiple biopsies taken from each of the following sites; the oesophagus, the body of stomach, the gastric antrum and the duodenum. The oesophagus was always biopsied prior to gastric biopsy to avoid contamination. ${ }^{13}$ Histological examination was by the Sydney system ${ }^{14}$ and all specimens were reported by a single histopathologist (MP). A rapid urease test was also performed. Patients subsequently had IgG ELISA antibodies and a European ${ }^{13} \mathrm{C}$-UBT performed. ${ }^{15}$ Using these methods, two groups were compared; those with either macroscopic or microscopic evidence of reflux oesophagitis

Helicobacter pylori: features

- Gram-negative, non-sporing, spiral bacillus

- urease enzyme protects it from acid attack in the stomach

- able to survive within macrophages

- infection probably childhood acquired and lifelong

- world-wide distribution and increasing in third world

- associated with gastritis, peptic ulcer disease and gastric carcinoma 
(the refluxers) and those who had macroscopically normal upper gastrointestinal tracts and no microscopic evidence of reflux oesophagitis (the non-refluxers). Statistical analysis was by $\chi^{2}$ tests (with Fisher's exact or Yates corrections as appropriate) and Student's $t$ tests (assuming unequal variance). Analyses were two-tailed, if appropriate, and $\mathrm{p}<0.05$ was taken as significant.

\section{Results}

A total of 114 patients (79 women, 35 men) were recruited over a nine-month period, average age was 78.9 years $( \pm 5.4)$. The main reasons for endoscopy were iron deficiency anaemia, reflux symptoms and epigastric pain accounting for over $60 \%$ of referrals.

Twenty-nine patients had macroscopic evidence of reflux oesophagitis (six of whom had histological features of reflux) and a further eight had histological evidence of reflux only, giving a total of 37 (table 1). They were compared to 33 patients who had neither macroscopic nor histological evidence of reflux oesophagitis as described above. In fact, the entire upper gastrointestinal tract of these 33 patients was normal. As expected more patients with evidence of reflux oesophagitis had heartburn as their primary symptom than in those without, $15 / 37$ vs $5 / 33, \mathrm{p}=0.03$. Of the 37 refluxers, $28(75.5 \%)$ were positive for $H$ pylori on at least one test compared to $27(81.8 \%)$ of the non-refluxers, $\mathrm{p}=0.7$.

Gastric histology was available on $33 / 37$ of the refluxers and $32 / 33$ of the non-refluxers. The incompleteness of the histological data was due to inadequate biopsy samples in some patients and inability to pass the scope into the stomach in the patients with oesophageal strictures. Twenty-four $(72.7 \%)$ of the refluxers had histological evidence of gastritis compared to $25(78.1 \%)$ of the non-refluxers, $p=0.8$. There was no significant difference in the site, severity or $H$ pylori positivity of the gastritis in the refluxers compared to the non-refluxers (table 2).

With respect to the non-invasive tests for $H$ pylori detection, the mean IgG ELISA antibody levels in the refluxers was 37.6 ( \pm 22.3 ) compared to $44.2( \pm 27.4)$ in the non-refluxers, $p=0.29$. Likewise the mean ${ }^{13} \mathrm{C}$-UBT levels were $13.3( \pm 17.2)$ in the

Table 1 Patients with reflux oesophagitis

\begin{tabular}{lcllll}
\hline \multirow{2}{*}{$\begin{array}{l}\text { Oesophagitis } \\
\text { grade }\end{array}$} & $n$ & \begin{tabular}{l} 
Other macroscopic findings \\
\cline { 3 - 6 } hernia
\end{tabular} & $\begin{array}{l}\text { Oesophagéal } \\
\text { stricture }\end{array}$ & Gastritis & Duodenitis \\
\hline Grade 1 & 11 & 4 & 0 & 2 & 1 \\
Grade 2 & 6 & 4 & 0 & 1 & 0 \\
Grade 3 & 8 & 2 & 0 & 1 & 0 \\
Grade 4 & 4 & 0 & $2^{\star}$ & 1 & 0 \\
Microscopic only & $8^{\star \star}$ & 2 & 0 & 0 & 1 \\
Total & 37 & 12 & 2 & 5 & 2 \\
\hline
\end{tabular}

^Both these patients had histological evidence of Barrett's oesophagus. ${ }^{\star}$ One patient with microscopic oesophagitis also had a gastric carcinoma.
Table 2 Histological findings and pattern of gastritis. There were no statistically signficant findings between the two groups

\begin{tabular}{lll}
\hline & $\begin{array}{l}\text { Reflux oesophagitis } \\
(n=37)^{\star}\end{array}$ & $\begin{array}{l}\text { Normal } \\
(n=33) \star \star\end{array}$ \\
\hline Gastritis & 24 & 25 \\
Antral & 7 & 5 \\
Body & 3 & 4 \\
Pan & 14 & 16 \\
& & 5 \\
Mild & 8 & 10 \\
Moderate & 9 & 10 \\
Severe & 7 & 16 \\
H pylori positive & 16 &
\end{tabular}

$\star$ This is the total number of patients with oesophagitis (macroscopic and microscopic). Histology available on $33 / 37$. ${ }^{\star \star}$ Histology available on $32 / 33$.

non-refluxers, $p=0.29$. Likewise the mean ${ }^{13} \mathrm{C}$-UBT levels were $13.3( \pm 17.2)$ in the refluxers compared to $14.3( \pm 13.4)$ in the non-refluxers, $\mathrm{p}=0.8$.

Twelve patients had macroscopic evidence of a hiatus hernia. Biopsies from oesophageal/ hernial sac mucosa of these patients revealed evidence of $H$ pylori in one patient while two others had histological evidence of Barrett's oesophagus; $H$ pylori was not identified in the oesophageal mucosa in either case.

There was no statistically significant difference in the numbers of patients on either aspirin or nonsteroidal anti-inflammatory drugs.

\section{Discussion}

The pathogenesis of inflammatory oesophageal diseases shows many similarities to the pathogenesis of gastritis and peptic ulcer disease and they have been considered as types of 'acidpeptic' disorders. ${ }^{16,17}$ Acid and pepsin have long been thought to be the major cause of mucosal damage caused by the reflux of gastric contents into the oesophagus, but other factors may also influence the severity of reflux oesophagitis, perhaps mucosal resistance, motility disorders ${ }^{18}$ and theoretically infectious agents. It is possible therefore that $H$ pylori may be involved in the pathogenesis of reflux oesophagitis.

Borkent and Baker demonstrated that colloid bismuth subcitrate together with cimetidine gave better results than cimetidine alone in healing ulcerative reflux oesophagitis. ${ }^{19}$ They also found that oesophagitis was accompanied by $H$ pylori infection in nine of 20 patients and concluded that $H$ pylori may have a role in the pathogenesis of reflux oesophagitis. This is supported by McCallum et al who suggested that patients with reflux oesophagitis had a higher incidence of $H$ pylori in the stomach. ${ }^{20}$ In their retrospective study of 21 consecutive patients with gastro-oesophageal reflux disease, $75 \%$ had gastritis and $60 \%$ had $H$ pylori identified in the antral biopsy specimens, significantly more than the findings in healthy controls $(10 \%$ and $5 \%$, respectively). They proposed that patients with gastro-oesophageal 
reflux disease may be at increased risk of colonisation by $H$ pylori as a result of raised intragastric $\mathrm{pH}$ with chronic antacid consumption, and also speculated that $H$ pylori may be involved in the pathophysiology of the disease. It is also interesting to note that Marshall and Warren's original study included 34 patients with abnormal oesophagi by endoscopic appearance and that $14(41 \%)$ of the 34 had $H$ pylori-like organisms identified in the antral biopsy specimens. ${ }^{21}$ However, more recent studies have found no such association between $H$ pylori and reflux oesophagitis. O'Connor and Cunnane prospectively studied 93 patients with reflux oesophagitis and found no association between $H$ pylori and grade of oesophagitis. ${ }^{22}$ Similarly, Coelho et al found that oesophageal infection was not significant in a group of 246 patients with a wide range of upper gastrointestinal tract disorders. They felt that oesophageal infection was simply due to gastrointestinal reflux. ${ }^{23}$

The present study is the first, to our knowledge, to investigate the possible association between $H$ pylori infection and reflux oesophagitis in elderly patients, who have a higher prevalence of infection. We found no convincing evidence for such an association. There was, as expected, a high overall rate of gastritis (over $75 \%$ ) in these patients but no statistically significant difference in the site, severity or $H$ pylori positivity of this gastritis when patients with evidence of reflux oesophagitis were compared to those who have macroscopically normal upper gastrointestinal tracts and microscopically normal oesophagi. Likewise ${ }^{13} \mathrm{C}$-UBT levels and IgG ELISA antibody levels were similar in both groups. We used multiple diagnostic modalities for $H$ pylori detection to maximise pick-up rates and minimise false negative results. Using these methods $H$ pylori was as likely to occur in those

1 Warren JR, Marshall BJ. Unidentified curved bacilli on gastric epithelium in active chronic gastritis. Lancet 1983; 1

2 Marshall BJ. Helicobacter pylori (review). Am f Gastroenterol 1994; 89: S1 16-S128.

3 The Eurogast Study Group. An international association between Helicobacter pylori infection and gastric cancer. Lancet 1993; 341: 1359-62.

4 Parsonnet J, Hansen S,. Rodriguez L, et al. Helicobacter pylori infection and gastric lymphoma. N Engl f Med 1994; 330: 1267-71.

5 Safe AF, Warren B, Corfield A, et al. Helicobacter pylori infection in elderly people: correlation between histology infection in elderly people: correlation betwe

6 O'Riordan TG, Tobin A, O'Morain C. Helicobacter pylor infection in elderly dyspeptic patients. Age Ageing 1991; 20: 189-92.

7 Wyatt JI, Shallcross TM, Crabtree JE, Heatley RV Helicobacter pylori, gastritis, and peptic ulceration in the elderly. F Clin Pathol 1992; 45: 1070-4

8 Webb PM, Knight T, Greaves S, et al. Relation between infection with Helicobacter pylori and living conditions in childhood: evidence for person to person transmission in early life. BMF 1994; 308: 750-3.

9 Banatvala N, Mayo K, Megraud F, Jennings R, Deeks JJ, Feldman RA. The cohort effect and Helicobacter pylori. $\mathcal{F}$ Infect Dis 1993; 168: 219-21.

10 Graham DY, Klein PD, Opekun AR, Boutton TW. Effect of age on the frequency of active campylobacter infection
diagnosed by the $\left[{ }^{13} \mathrm{C}\right]$ urea breath test in normal subjects and patients with peptic ulcer disease. $\mathcal{F}$ Infect Dis 1988; 157: and patients

11 Graham DY, Adam E, Klein PD, et al. Epidemiology of Campylobacter pylori infection. Gastroenterol Clin Biol 1989; 13: 84B-89B.

12 Stolte M, Bethke B. Elimination of Helicobacter pylori under treatment with omeprazole. $Z$ Gastroenterol 1990; 28
271-4.

\section{Summary/learning points}

- lack of data regarding $H$ pylori and reflux oesophagitis

- elderly patients have high prevalence of life-long $H$ pylor $i$ infection

- elderly are ideal group to study $H$ pylori induced mucosal changes

- $75 \%$ of elderly with reflux oesophagitis have $H$ pylori infection

- $80 \%$ without reflux oesophagitis have $H$ pylori infection

- no differences exist in the site, severity or $H$ pylori positivity of gastritis in elderly with and without reflux oesophagitis

- there is no evidence for a role for $H$ pylori in the pathogenesis of reflux oesophagitis

with no evidence of reflux as in those with definite evidence.

Only two of our patients had evidence of Barrett's oesophagus and in neither of these was $H$ pylori detected in the mucosa. This is in keeping with the work of Paull and Yardley who found no direct association between $H$ pylori and Barrett's oesophagus in a group of 26 patients with the condition. ${ }^{24}$ Other authors have reached similar conclusions. ${ }^{22}$

We conclude that extensive investigation of a group of elderly patients with a high overall level of $H$ pylori infection has revealed no evidence of an association between $H$ pylor $i$ and reflux oesophagitis. The high prevalence of $H$ pylori infection in patients with evidence of reflux can be fully explained by the presence of incidental gastritis.

We would like to acknowledge Dilip Patel and Mohammed Arif Mirza (medical students) who helped in the preliminary stages of this study.

13 Agnholt J, Fallingborg J, Moller-Petersen J, et al. The occurrence of Helicobacter pylori in the oesophagus. Eur $\mathcal{F}$ Gastroenterol Hepatol 1991; 3: 685-8.

14 Price AB. The Sydney system: histological division. $f$ Gastroenterol Hepatol 1991; 6: 209-22.

15 Logan RPH, Dill S, Bauer FE, et al. The European ${ }^{13} \mathrm{C}$-urea breath test for the detection of Helicobacter pylori. Eur $\mathcal{F}$ Gastroenterol Hepatol 1991; 3: 915-21.

16 Hirschowitz BI. A critical analysis, with appropriate controls, of gastric and pepsin secretion in clinical oesophagitis. Gastroenterology 1991; 101: 1149-58.

17 Bateson MC, Bouchier IA. The association of oesophagitis with disease in the stomach and duodenum. Postgrad Med $\mathcal{J}$ with disease in the

18 Baldi F, Corinaldesi R, Ferrarini F. Gastric secretion and emptying of liquids in reflux oesophagitis. Dig Dis Sci 1981 26: 886-9.

19 Borkent MV, Beker JA. Treatment of ulcerative reflux oesophagitis with colloidal bismuth subcitrate in combination with cimetidine. Gut 1988; 29: 385-9.

20 McCallum RW, DeLuca V, Marshall BJ, Prakash LC Prevalence of campylobacter-like organisms in patients with gastro-esophageal reflux disease versus normals. Gastroenterology 1987; 92: 1524 (abstract).

21 Marshall BJ, Warren JR. Unidentified curved bacilli in the stomach of patients with gastritis and peptic ulceration. Lancet 1984; $1: 1311-4$.

22 O'Connor HJ, Cunnane K. Helicobacter pylori and gastrooesophageal reflux disease, a prospective study. Ir $7 \mathrm{Med} S c i$ 1994; 163: 369-73.

23 Coelho LGV, Das SS, Payne A, Karim QN, Baron JH, Walker MM. Campylobacter pylori in esophagus, antrum and duodenum. Dig Dis Sci 1989; 34: 445-8.

24 Paull G, Yardley JH. Gastric and esophageal campylobacter in patients with Barrett's esophagus. Gastroenterology 1988 ,
95: 216-8. 